\title{
Association Rule Mining Based Extraction of Semantic Relations Using Markov Logic Network
}

\author{
K.Karthikeyan ${ }^{1}$ and Dr.V.Karthikeyani ${ }^{2}$ \\ ${ }^{1}$ Research and Development Centre, Bharathiar University, Coimbatore, Tamil Nadu \\ ${ }^{2}$ Department of Computer Science, Thiruvalluvar Govt. Arts College, Rasipuram, \\ TamilNadu
}

\begin{abstract}
Ontology may be a conceptualization of a website into a human understandable, however machinereadable format consisting of entities, attributes, relationships and axioms. Ontologies formalize the intentional aspects of a site, whereas the denotative part is provided by a mental object that contains assertions about instances of concepts and relations. Semantic relation it might be potential to extract the whole family-tree of a outstanding personality employing a resource like Wikipedia. In a way, relations describe the linguistics relationships among the entities involve that is beneficial for a higher understanding of human language. The relation can be identified from the result of concept hierarchy extraction. The existing ontology learning process only produces the result of concept hierarchy extraction. It does not produce the semantic relation between the concepts. Here, we have to do the process of constructing the predicates and also first order logic formula. Here, also find the inference and learning weights using Markov Logic Network. To improve the relation of every input and also improve the relation between the contents we have to propose the concept of ARSRE. This method can find the frequent items between concepts and converting the extensibility of existing lightweight ontologies to formal one. The experimental results can produce the good extraction of semantic relations compared to state-of-art method.
\end{abstract}

\section{KEYWORDS}

Ontology, Semantic Web, Markov Logic Network, Semantic Relation, Association Rule Mining.

\section{INTRODUCTION}

Ontology is a specification of conceptualization it specifies the meanings of the symbols in an information systems. This ontology has three kinds of components like individuals, classes and properties. Individuals are nothing but the things or objects in the world. Classes are a set of individuals. Properties are between individual and their values. Ontology learning things about with information discovery numerous knowledge [4] sources associated with its illustration through an onto logic structure and together with metaphysics population, constitutes associate approach for automating the information acquisition method. Ontology may be an illustration of information formal. It offer a transparent and consistent illustration of nomenclature and ways that facilitate individuals to watch issues and managing affairs, offer public vocabulary of areas and outline totally different levels of formal meanings of terms and relationship between terms.

Ontologies square measure the backbone of the linguistics internet also as of a growing range of knowledge-based systems. A long standing goal of Artificial Intelligence is to create an autonomous agent which will scan and understand text. The method of meta-physics learning from text includes three core subtasks. There are learning of the ideas which will represent the meta-physics, learning of the linguistics relations among these ideas and at last one is learning of 
a set of abstract through rules. Ontology learning tools discover binary relations not just for lexical things however additionally for ontological ideas. Ontology learning is bothered with data acquisition and within the context of this volume additional specifically with data acquisition from text. Ontology learning is inherently multi-disciplinary attributable to its robust affiliation with the semantic web.

In ontology learning [9], [10], [11], [26] had two kinds of evaluation procedures. There are manual evaluation and posteriori evaluation. Manual evaluation has an advantage which is supposed to know the concepts and their relationships in their domain of expertise, they are supposedly able to tell either a given ontology represents a domain or not. The manual evaluation is subjective and time consuming. The posteriori evaluation also same like manual evaluation but small change in that it is more time consuming and represent the evaluation properly. The grammatical relations are arranged in a hierarchy, rooted with the most generic relation, dependent. When the relation between a head and its dependent can be identified more precisely, relations further down in the hierarchy can be used.

Ontologies became omnipresent in current generation information systems. Associate metaphysics is associate explicit, formal illustration of the entities and relationships which will exist during a domain of application. The term meta-physics came from philosophy and was applied to data systems within it have to characterize the formalization of a body of information describing a given domain. One amongst the key drivers for the popularity of that idea was the conclusion that many of the foremost difficult issues within the data technology field, cannot be resolved while not considering the linguistics intrinsically embedded in every explicit data system.

Ontology learning uses [11] methods from a diverse spectrum of fields such as machine learning, knowledge acquisition, natural language processing, information retrieval, artificial intelligence, reasoning, and database management. Ontology learning framework for the Semantic Web that included ontology importation, extraction, pruning, refinement, and evaluation giving the ontology engineers a wealth of coordinated tools for ontology modeling.

Semantic ability [22] means that not simply that two distinct knowledge systems are ready to exchange information during a given format, that the token should have an equivalent which means for each parties. Customary illustration languages for ontologies like the raptorial bird net meta-physics language and publicly obtainable ontologies will greatly facilitate the event of practical systems, but the process of desegregation and reusing ontologies remains fraught with issue. Strategies for machine-controlled discovery of information and construction of ontologies will facilitate to beat the tedium and mitigate non-compliance however gaps and inconsistencies are inescapable.

Learning semantic [6] resources from text instead of manually creating them might be dangerous in terms of correctness, but has undeniable advantages: Creating resources for text processing from the texts to be processed will fit the semantic component neatly and directly to them, which will never be possible with general-purpose resources. Further, the cost per entry is greatly reduced, giving rise to much larger resources than an advocate of a manual approach could ever afford.

Semantic annotation[19] of a corpus will be performed semi-automatically by varied annotation tools that speed up the entire procedure by providing a friendly interface to a website knowledgeable. A manually annotated corpus will be wont to train associate degree info extraction system. The aim of those approaches is that the exploitation of associate degree initial small-sized lexicon and a machine learning-based system for the lexicon enrichment through associate degree repetitive approach. 
International Journal of Web \& Semantic Technology (IJWesT) Vol.5, No.4, October 2014

Producing robust components to process human language as part of applications software requires attention to the engineering aspects of their construction. For that purpose we have to use the GATE (General Architecture of Text Engineering) tool. This tool [27] is used to perform the dataset progress procedure. According to this tool we had to load our xml dataset. This could be processed to generate the input files for further processing. This GTAE tool shall be used in initial stage of ontology process. The ontology learning process mainly focused this kind of tool for enlarge the ontology process. The framework provides a reusable design for an LE software system and a set of prefabricated software building blocks. As a development process it helps its users to minimize the time they spend building new LE systems or modifying existing ones, by aiding overall development and providing a debugging mechanism for new modules.

The GATE framework contains a core library and a set of reusable lupus modules. The framework implements the design and provides facilities for process and visualizing resources, together with illustration, import and export of information. GATE element is also enforced by a spread of programming languages and databases, however in every case they are delineate to the system as a Java category.

Statistical Relative Learning (SRL) focuses [2] on domains wherever knowledge points square measure not freelance and identically distributed. It combines ideas from applied mathematics learning and inductive logic programming and interest in its adult rapidly. One in every of the foremost powerful representations for SRL is Markov logic, that generalizes each markov logic random fields and first-order logic.

Weight Learning in Markov logic could be a bell-shaped improvement drawback, and therefore gradient descent is absolute to realize the worldwide optimum. However, convergence to the present optimum is also very slow. MLN's [3] square measure exponential models, and their decent statistics square measure the number of times every clause is true within the information. The Markov Logic random fields [12] computing the chance in MLNs needs computing the partition functions, that is mostly stubborn. This makes it troublesome to use ways that require activity line searches, that involve computing the perform as well as gradient.

Wordnet was accustomed notice nouns that area unit derived from verbs and to filtrate words that are not in the noun information. Noun in wordnet area unit joined to their derivationally connected verbs, however there is no indication regarding that springs from that. WordNet, that uses documents retrieved from the Web. However, in their approach, the query strategy is not entirely satisfactory in retrieving relevant documents which affects the quality and performance of the topic signatures and clusters. Using Word Net to enrich vocabulary for ontology domain, we have presented the lexical expansion from Wordnet approach providing a method of accurately extract new vocabulary for an ontology for any domain covered by WordNet.

The vocabulary of associate degree object language for a given domain consists of names representing the people of the domain, predicates standing for properties and relations and of logical constants.

The meaning of a predicate is in general not independent of the meaning of other predicates. This interdependence is expressed by axioms and intensional and extensional definitions. An extensional definition of a predicate is simply the list of the names of the individuals that constitute its extension. An intensional definition of a predicate (definiendum) is the conjunction of atomic sentences (definientia) stating the properties an individual must possess for the predicate to apply. Ontology for an object language is a non-logical vocabulary supplemented by a set of 
- axioms

- intensional definitions

- extensional definitions

The axioms picture structural properties of the domain and limit the possible interpretation of the primary terms. The intensional and extensional definitions are terminological.

There are containing two ways that conceiving ontology construction, very cheap up predominant within the methodology of arithmetic and a prime down approach that's predominant disciplines wherever the domain consists of objects of the planet a given as in science.

Alchemy is a software package [13] providing a series of algorithms for statistical relational learning and probabilistic logic inference, based on the Markov logic representation. Alchemy may be a computer code tool designed for a large varies of users. It assumes the reader has noises of classical machine learning algorithms and tasks and is accustomed to first-order and markov logic and a few likelihood theory. Alchemy may be a add progress and is frequently being extended to satisfy these desires.

During weight learning every formulas [15] are reborn to conjunctive traditional form (CNF) and a weight is learned for every of its clauses. If a formula is preceded by a weight within the inpu.mln file, the load is split equally among the formula's clauses. The load of a clause is employed because the mean of a Gaussian previous for the learned weight.

This paper [8] told Anaphora resolution system. This anaphora lets be the front step of Natural Language Processing (NLP). Understanding the Text means, understanding the meaning of context or concept. Anaphora system produces most likelihood antecedent after development of machine learning approach. The knowledge poor strategy provides best results compare to previous knowledge rich strategy. The computational strategy provide maximum share to produce most accurate antecedent. But not least, preprocess task is base for computational strategy perform well good manner.

The name of animal or human being is more concentrate in AR community to categorization of candidate set. It is easy to accept or reject the discourse in candidate set. So, we concentrate much effort to be taken against to recognize the animacy agreement. But this system constructed rule based AR system.

Learning from text and natural language is one of the great challenges of Artificial Intelligence and machine learning. The Probabilistic Latent Semantic Analysis (PLSA) [7] is a statistical model which has been called as Aspect Model. The aspect model is a latent variable model for co-occurrence data which associate an unobserved class variable. The PLSA model has an advantage of the well established statistical theory for model selection and complexity control to determine the optimal number of latent space dimensions.

Commonsense knowledge parsing can be performed using a combination of syntax and semantics, via syntax alone (making use of phrase structure grammars), or statistically, using classifiers based on training algorithms. Probabilistic inference aims at determining the probability of a formula given a set of constants and, maybe, other formulas as evidence. The probability of a formula is the sum of the probabilities of the worlds where it holds.

Ontology learning can be dividing the portion of relationship mechanism. This process called as semantic relation extraction. This kind of ontology learning process usually takes the steps of finding weight learning. The first process behind in this stage is to construct the predicates and 
first order logic formula. A first order logic formula can be constructing from the first order logic knowledge base using Markov Logic Network. According to the predicates we have to build the MLN file. After that build the training file and also the test files. These all based on the input we are taking at the time initialization. Here, we have to find the inference values and last predicated number etc., and then we have to learn the parameter weights of each and every document progressed in our corpus.

As a summary, the main contributions of our work include the following:

i. We present the novel methodology of Ontology Based Semantic Relation Extraction to find the frequent items relationship based on association rule mining.

ii. We present the construction of predicates and first order logic and weight learning processes based on Markov Logic Networks.

iii. Then we present the avoidance of non useful words and also redundant words and make the tree structure using thesaurus.

The rest of this paper is organized as follows: Section 2 reviews the related work. Section 3 contains the process of General Markov Logic Network details. Section 4 formulates the proposed framework of Association Rule Mining Based Semantic Relation Extraction. In further section it will be called as ARSRE, gives results of the semantic relation extraction. Section 5 has the experimental results for the projects. Section 6 formulates the Conclusion for the projects and Section 7 contains the Future enhancement of the projects. Thus the paper clearly explains the concept of the ARSRE.

\section{RELATED WORK 2.1 Ontology Learning}

Ontology learning will be outlined because the set of methods and techniques used for building metaphysics from scratch, enriching or adapting associate in nursing existing meta-physics during semi-automatic fashion victimization many sources. Ontology may [10], [26] be an illustration of information formal. It offer a transparent and consistent illustration of nomenclature and ways that facilitate individuals to watch issues and managing affairs, offer public vocabulary of areas and outline totally different levels of formal meanings of terms and relationship between terms. The meta-physics will be considered as vocabulary of terms and relationships between those terms during a given domain. Ontology learning use strategies from a various spectrum of fields like machine learning, information acquisition, natural language process, information retrieval, computer science, reasoning and database management.

\subsection{Semantic Web}

Semantic internet technologies are aimed toward providing the mandatory representation languages and tools to bring linguistics to the current internet contents. The semantic Meta data [5], [16] can be producing the language of Resource Description language. The RDF we are having three kinds of elements. There are resources, literals and properties. The resources are also called as web objects. The web objects are identified through URI. Literals are the atomic values it contain the values of strings, dates and numbers. Properties also identified through URI. Properties are the binary relationship between resources and literals. The linguistics internet offers the potential for facilitate, permitting a lot of intelligent search queries supported websites marked up with linguistics data. 
International Journal of Web \& Semantic Technology (IJWesT) Vol.5, No.4, October 2014

\subsection{Semantic Relation}

Semantic relation [6] it might be potential to extract the whole family-tree of an outstanding personality employing a resource like Wikipedia. In a way, relations describe the linguistics a relationship among the entities involves that is beneficial for a higher understanding of human language. Semantic relations square measure a vital component within the construction of ontologies and models of downside domains.

To express the relation we are attempting to accumulate information regarding lexical and alternative linguistics relations aside from similarity or synonymy by distinctive syntactical constructions. This paper [28], [14] presents discovering the semantic relations between the ontology concepts. Here this could be divided into two kinds of concepts. There high level concepts and low level concepts. The discovery process isn't supported the belief that verbs typically indicate semantic relation between ideas and doesn't exploit lexicon-syntactic patterns or cluster ways or any external data sources like wordnet. Discovering semantic relation between high level concepts based on annotation. In this approach, for each document we search for the different pairs of concepts that have overlapping instances. We assume that abstraction overlap between conception instances within the text implies a semantic relationship. In final part of in this high level concepts we simply apply applied math methods to document information that's to the placement of conception instances in text. To discover the semantic relation [19] between low-level concepts we have to apply the variation on the definition of the instance offset of each low level concept. Using a named entity recognizer for the annotation of low-level ideas instances we will be ready to any automize the applying of the projected variation to get linguistics relations between low-level ideas. Semantic relation [29] it might be potential to extract the whole family-tree of a outstanding personality employing a resource like Wikipedia. In a way, relations describe the linguistics a relationship among the entities involves that is beneficial for a higher understanding of human language. Semantic relations square measure a vital component within the construction of ontologies and models of downside domains.

\subsection{Association Rule Mining}

Association rules [18] square measure supported the notion of group action that is an observation of the co-occurrence of a collection of things. Once handling southwest information, the most challenge consists in distinguishing interesting transactions and things from the semi-structured and heterogeneous nature of that information. During this means, it becomes crucial to use the maximum amount as potential the data provide by the ontologies so transactions will be simply mined. The basic task of association data processing is to seek out all association rules with support and confidence bigger than user fixed minimum support and minimum confidence threshold values. Association rule mining is taken into account jointly of the foremost vital tasks in data discovery in databases. Association rule mining employs the support live, that treats dealings equally. In contrast, totally different transactions have different weights in datasets. Typically association rule square measure generated ranging from frequent item sets generated from frequent item sets. The generalized association rule learning algorithmic program extends its baseline by aiming at descriptions at the appropriate taxonomy level.

\subsection{Syntactic Structure Features}

A Syntactic Analysis [20] is useful because, it provides a hierarchical set of groupings words and phrases which can be the basis for a general-purpose, finite and compositional procedure to extract meaning from any sentence of the language. A syntactic analysis tells us that phrases. It could be argued that it is impossible to talk of a natural grouping without considering meaning. Syntactic structures[24] that reflect these groupings of items into larger meaningful units. This 
International Journal of Web \& Semantic Technology (IJWesT) Vol.5, No.4, October 2014

idea of compositional semantics is very widespread, and it is one of the guidelines which will be adopted here in deciding on suitable syntactic structures. Establishing the grammar structure of resultatives, we tend to return to a more general conclusion regarding the connection between the two levels of grammatical representation. It is necessary to grasp that the mnemotechnic names given to those classes area unit basically whimsical, because it is that the approach that the labels area unit employed in the principles and within the lexicon that offers significance to them. Syntactic approaches use similarity considering the relationships between predicate and argument.

\subsection{First-Order Logic}

A first order knowledge base [15] could be a set of sentences or formulas in first-order logic. Formulas area unit created victimization four kinds of symbols. There are constants, variables, functions and predicates. Constant symbols represent objects within the domain of interest. Variable symbols vary over the objects within the domain. Operate symbols represent mappings from tuples of objects to things. Predicate symbols represent relations among objects within the domain or attributes of objects. A first-order computer memory unit is often seen as worlds, if a world violates even one formula, its zero likelihood. The basic idea in markov logic is to melt these constraints. Once a world violates one formula within the computer memory unit it's less probable, however not possible.

Our work is also related with some existing support of predicates based markov logic network. This process inputs are taken comes under the progress of concept hierarchy extraction. This could have the results of web based information of every document. The performance of this work related to the semantic relation. The authors [15] explore the issues of semantic relation extraction. So, our goal is to improve the semantic relation extraction process based on Markov Logic Networks. To employ our process, before performing the semantic relation extraction we do some progress of construction of predicate and first-order logic formula. After that we perform the process of finding inference and weight learning. For finding the inference we use Markov Logic Network. For weight learning we can use the formulas and also database of atoms for calculates the weights. Learning rate can be used to perform this kind of task.

As a final point, there are some works adopt to improve the semantic relation extraction. Association rule mining mainly used to find the relationship between the frequent item sets. Next, conversion process is to the extensibility of existing lightweight ontologies to formal one. These processes are performing by the proposed techniques of named as ARSRE. This method that provides the effective and efficient results.

\section{MARKOV LOGiC NetWORK}

Markov Logic [17] is indistinguishable from first-order logic, except that every formula includes a weight connected. Markov logic may be a straightforward nonetheless powerful combination of markov networks and first-order logic. Markov logic raises the quality of markov networks to encompass first-order logic. Recall that a first-order domain is defined by a collection of constants representing objects within the domain and a collection of predicates representing properties of these objects and relations between them. A predicate are often grounded by replacement its variables with constants. A first-order Knowledge Base (KB) may be a set of formulas in first-order logic, created from predicates victimization logical connectives and quantifiers. A formula in markov logic may be a formula in first-order logic with associate degree associated weight. The basic plan in markov logic is to melt these constraints. When a world violates one formula within the $\mathrm{K}$ it's less probable, however not possible. Less formulas a world 
violates, a lot of probable it's. Associate degree MLN [1] is often viewed as a template for constructing markov networks. In different worlds it will manufacture different networks and this also has wide varied size, however all can have certain regularities in structure and parameters, given by the MLN.

Combining chance and first-order logic in a very single illustration has long been a goal of Artificial Intelligence. Probabilistic graphical models change us to efficiently handle uncertainty. First-order logic permits us to succinctly represent a good variety of information.

A Markov Logic network [21] may be a first-order knowledge domain with a weight attached to every formula, and may be viewed as a temple for constructing Markov Networks. MLNs give a compact language to specify terribly giant a Markov Networks, and therefore the ability to flexibly and modularly incorporate a large vary of domain information into them. Several necessary tasks in applied mathematics relative learning, like collective classification, link prediction, link-based cluster, social network modeling, and object identification, area unit naturally developed as instances of MLN learning and illation.

Markov Logic, linguistics internet languages can be created probabilistic just by adding weights to statements and linguistics web illation engines may be extended to perform probabilistic reasoning merely by passing the proof of Directed Acyclic Graph (DAG), with weights connected, to a probabilistic illation system. Weights is also set by hand inferred varied sources or learned mechanically from information. MLN acts as a template for a markov network. We have extended and adapted several of these standard methods to take particular advantage of the logical structure in a markov logic network, yielding tremendous savings in memory and time. Markov logic combines first-order logic and probabilistic graphical models in a unifying representation. The main idea behind Markov Logic is that, unlike first-order logic, a world that violates a formula is not invalid, but only less probable.

Statistical Relative learning combines [2] the communicatory power of data representation formalisms with probabilistic learning approaches, therefore enabling one to represent grammar dependencies between words and capturing applied mathematics information of words in text. The markov logic network represent an approach for applied mathematics relative learning that mixes first order logic with markov random fields. Associate MLN could be a first order logic mental object weights, which may be either positive or negative, associated to every formula.

The main idea behind Markov logic [23] is that, unlike first-order logic, a world that violates a formula is not invalid, but only less probable. This is done by attaching weights to first-order logic formulas: the higher the weight, the bigger is the difference between a world that satisfies the formula and one that does not, other things been equal.

Two common inference tasks in Markov Logic are the maximum a posteriori (MAP) and probabilistic inference. MAP inference aims at finding the most probable state of the world given some evidence. In Markov Logic this task is the same as finding the truth assignment that maximizes the sum of the weights of satisfied formulas. This can be done by any weighted satisfiability solver. Probabilistic inference aims at determining the probability of a formula given a set of constants and, maybe, other formulas as evidence. The probability of a formula is the sum of the probabilities of the worlds where it holds. There are two approaches for learning the weights of a given set of formulas. There are generative and discriminative learning. Generative learning aims at maximizing the joint likelihood of all predicates while discriminative, at maximizing the conditional likelihood of the query predicates given the evidence ones. Maximum-likelihood or MAP estimates of Markov network weights cannot be computed in closed form, but, because the log-likelihood is a concave function of the weights, they can be found efficiently using standard gradient based or quasi-Newton optimization methods 
A term is any expression representing an object in the domain. It can be a constant, a variable, or a function applied to a tuple of terms. For example, Anna, x, and GreatestCommonDivisor(x, y) are terms. An atomic formula or atom is a predicate symbol applied to a tuple of terms (e.g., Friends(x, Mother of (Anna))).

Parentheses may be used to enforce precedence. A positive literal is an atomic formula; a negative literal is a negated atomic formula. The formulas in a KB are implicitly conjoined, and thus a KB can be viewed as a single large formula. A ground term is a term containing no variables. A ground atom or ground predicate is an atomic formula all of whose arguments are ground terms. A possible world or her brand interpretation assigns a truth value to each possible ground atom.

The syntax of the formulas in an MLN is the standard syntax of first-order logic. An MLN can be viewed as a template for constructing Markov networks. Given different sets of constants, it will produce different networks, and these may be of widely varying size, but all will have certain regularities in structure and parameters, given by the MLN. The Probability distribution over possible worlds $\mathrm{x}$ specified by the ground markov network. It can be represented by

$$
P(X=x)=\frac{1}{Z} \exp \left(\sum_{i} w_{i} n_{i}(x)\right)=\frac{1}{Z} \prod_{i} \phi_{i}\left(x_{\{i\}}\right)^{n_{i}(x)}
$$

We defined MLNs as log-linear models, they could equally well be defined as products of potential functions, as the second equality above shows. This will be the most convenient approach in domains with a mixture of hard and soft constraints. The graphical structure of markov network follows from there is an edge between two nodes of markov network iff the corresponding ground atoms appear together in at least one grounding of one formula in L. Thus, the atoms in each ground formula form a (not necessarily maximal) clique in markov network.

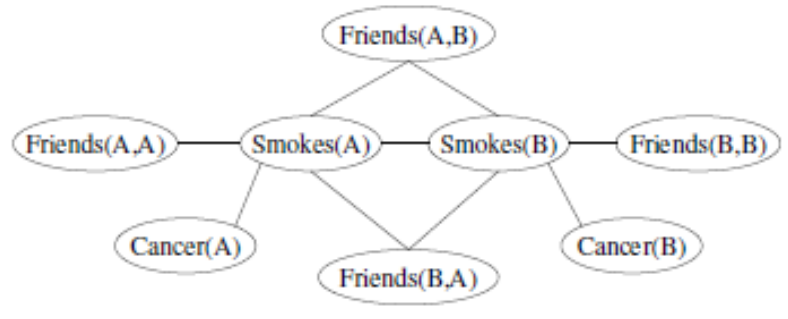

Figure 1 Ground Markov Network

A first-order KB can be seen as a set of hard constraints on the set of possible worlds, if a world violates even one formula, it has zero probability. The basic idea in MLNs is to soften these constraints: when a world violates one formula in the KB it is less probable, but not impossible. The fewer formulas a world violates, the more probable it is. Each formula has an associated weight that reflects how strong a constraint it is the higher the weight, the greater the difference in $\log$ probability between a world that satisfies the formula and one that does not, other things being equal. In an MLN, the derivative of the negative conditional log-likelihood (CLL)

with respect to a weight is the difference of the expected number of true groundings of the corresponding clause and the actual number according to the data.

This last assumption allows us to replace functions by their values when grounding formulas. Thus the only ground atoms that need to be considered are those having constants as arguments. 
Features can also be learned from data, for example by greedily constructing conjunctions of atomic features.

\section{ARSRE (Association Rule Mining Based Semantic Relation EXTRACTION)}

ARSRE is an Association Rule Mining Based Semantic Relation Extraction. The ARSRE describes the process of semantic relation extraction. Semantic relation is the process that contains sub process of construction of predicates and first order logic formula, Finding Inference and weight. The most existing technique of semantic relation extraction was developed from syntactic word features and markov logic networks only. In our proposed system semantic relation extraction based on finding frequent items relationship and avoidance of non useful words. Frequent items should be found with the help of association rule mining. The ARSRE techniques have some types. There are Construction of first order logic predicates, finding inference and weigh, semantic relation extraction and also avoidance of non useful words. The OBCHED process clearly explains in figure 2 .

\subsection{Construction of First Order Logic Predicates}

Here, we are going to handle the process of first order logic predicates. For that purpose we have to take the methods of two kinds. One is selection of predicates based on word features. This word features contains the process of extraction of domain concepts, instances, attributes, values an also the relationships. This kind of semantic relation extraction process we choose the choice of features to consider the word features and punctuation features, such as "The elevation of Fuxian Lake is 1722.5 meters", which the word "elevation" means

"Fuxian Lake" and "1722.5 meters" is the R (I, V) and the punctuation mark ":" can also reflect the $\mathrm{R}(\mathrm{C}, \mathrm{I})$. For finding the predicates based on word features we have to use the formula like

HasWord $(+w, s)^{\wedge}$ HasEntity $(+e 1, s)^{\wedge}$ HasEntity $(+e 2, s)=>$ HasRelation $(+r, e 1, e 2)$

Second one is selection of predicates based on syntactic features. In one sentence we are having more than three entities at that time we do not take the word feature selection it's automatically move onto the syntactic feature selection. For describing the syntactic feature selection we can the example of "Yunnan ethnic village and Daguanlou lie between water, covers an area of 89 hectares", it has two entity "Yunnan ethnic village", "Daguanlou", and a value entity "89 hectares", the characteristic "area" is described in "Yunnan ethnic village" or "Daguanlou", during the entity relationship matching, it is easy to make mistake. For avoiding this kind of mistake we generate the formula such as,

$$
\text { BigThan (Distance (e1, e3), Distance }(e 2, e 3))^{\wedge} \text { HasWord }(+w, s)=>\text { HasRelation }(+r, \text { e1,e3) }
$$

Here, the entity of e1 and e2 has the parallel relationship. According to this relationship we have to find the first order logic predicates. So we can express the formula like

Parallel $(e 1, e 2)^{\wedge}$ HasRelation $(r, e 1, e 3)=>\operatorname{HasRelation}(r, e 2, e 3)$

The entity 1 and entity 2 has the relationship of parallel. According to this kind of processes we have to construct the first order predicates.

\subsection{Inference and Weight Learning}

The process of finding inference can be done with the help of markov logic network. The MLNs inference including maximum likelihood inference calculates the marginal probabilities and 
International Journal of Web \& Semantic Technology (IJWesT) Vol.5, No.4, October 2014

calculates the conditional probability. Maximum likelihood reasoning process can be expressed a predicate formula $\mathrm{x}$ and find the relative formula $\mathrm{y}$ which can be express the probability as

$$
\max _{y} P(y / x)
$$

Then according to the markov logic network's joint probability formula it can be transformed to

$$
\max _{y} \sum_{i} w_{i} n_{i}(x, y)
$$

According to this transformation we have to find the inference value for each every document in the corpus.

Next, we do the process of weight learning. For finding weight learning we have to take the discriminative learning based MLN method. Then we assume the evidence predicate $\mathrm{x}$ and query predicate $\mathrm{y}$, this could be the large collections of y produce the probability condition of,

$$
p_{w}(y \mid x)=\frac{1}{Z_{x}} \exp \left(\sum_{i \in F_{r}} \omega_{i} n_{i}(x, y)\right)
$$

Here, we take the greater than true value number. According to that find the weights in the learning process.

Originally proposed learning weights generatively using pseudo-likelihood. Pseudo-likelihood is the product of the conditional likelihood of each variable given the values of its neighbors in the data. While efficient for learning, it can give poor results when long chains of inference are required at query time. Pseudo-likelihood is consistently outperformed by discriminative training, which minimizes the negative conditional likelihood of the query predicates given the evidence ones.

In an MLN, the derivative of the negative conditional log-likelihood (CLL) with respect to a weight is the difference of the expected number of true groundings of the corresponding clause and the actual number according to the data. Use this approach and discuss how the resulting method can be viewed as approximately optimizing log-likelihood. However, the use of discriminative MLNs is potentially complicated by the fact that the MPE state may no longer be unique. 


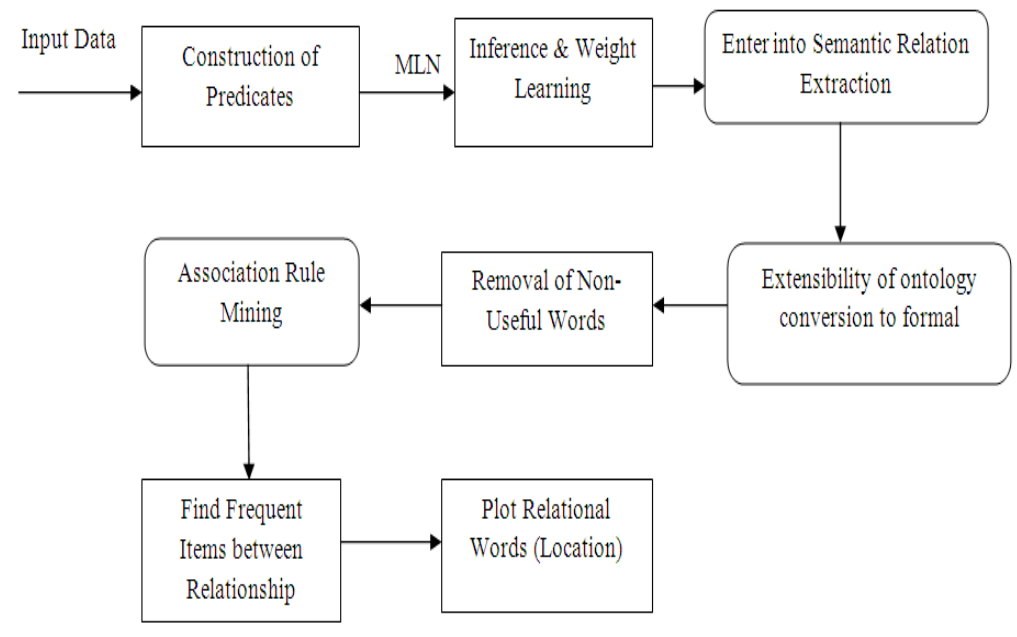

Figure 2. ARSRE Process

\subsection{Association Rule Mining}

The Association rule mining process is one of the contributions of our proposed system work. This association rule mining is used to find the relationship between the frequent items. Here, we take the input from semantic web data. The problem of discovering association rules can be formally described as follows. Let $\mathrm{I}=\{\mathrm{i} 1, \mathrm{i} 2 \ldots \mathrm{im}\}$ be a set of $\mathrm{m}$ literals, called items. Let $\mathrm{D}=$ $\{\mathrm{t} 1, \mathrm{t} 2$. . t $\mathrm{tn}\}$ be a database of $\mathrm{n}$ transactions where each transaction is a subset of $\mathrm{I}$. An itemset is a subset of items. The support of an itemset $S$, denoted by $\sup (X)$, is the percentage of transactions in the database D that contain S. An itemset is called frequent if its support is greater than or equal to a user specified threshold value. An association rule $r$ is a rule of the form $X=>Y$ where both $\mathrm{X}$ and $\mathrm{Y}$ are non-empty subsets of $\mathrm{I}$ and $\mathrm{X} \cap \mathrm{Y}$. $\mathrm{X}$ is the antecedent of $\mathrm{r}$ and $\mathrm{Y}$ is called its consequent. The support and confidence of the association rule $\mathrm{r}$ : $\mathrm{X}=>\mathrm{Y}$ are denoted by $\sup (\mathrm{r})$ and $\operatorname{conf}(\mathrm{r})$. These measures can be interpreted in terms of estimated probabilities as $\sup (\mathrm{r})$ $=\mathrm{P}(\mathrm{X} \cap \mathrm{Y})$ and $\operatorname{conf}(\mathrm{r})=\mathrm{P}(\mathrm{Y} \mid \mathrm{X})$ respectively. Another interesting measure is the lift or interest factor of a rule, denoted by lift(r), which computes the ratio between the rule probability and the joint probability of $\mathrm{X}$ and $\mathrm{Y}$ assuming they are independent, that is, $\mathrm{P}(\mathrm{X}[\cap \mathrm{Y}) /(\mathrm{P}(\mathrm{X}) \mathrm{XP}(\mathrm{Y}))$. If this value is 1 , then $\mathrm{X}$ and $\mathrm{Y}$ are independent. The higher this value, the more likely that the existence of $\mathrm{X}$ and $\mathrm{Y}$ together in a transaction is not just a random occurrence, but because of some relationship between them.

The basic task of association data mining is to find all association rules with support and confidence greater than user specified minimum support and minimum confidence threshold values. Mining association rules basically consists of two phases. One is compute the frequent itemsets with respect to the minimum support, and another one is three kinds of elements. There are resources, literals, and properties. Resources are web objects (entities) that are identified through a URI, literals are atomic values such as strings, dates, numbers, etc., and properties are binary relationships between resources and literals. Properties are also identified through URIs. The basic building block of RDF is the triple: a binary relationship between two resources or between a resource and a literal. The resulting metadata can be seen as a graph where nodes are resources and literals, and edges are properties connecting them. RDFS extends RDF by allowing triples to be defined over classes and properties. In this way, we can describe the schema that rules our metadata within the same description framework. Later on, the ontology web language 
International Journal of Web \& Semantic Technology (IJWesT) Vol.5, No.4, October 2014

(OWL) was proposed to provide well-founded logic inferences over semantic descriptions. Most sublanguages of OWL are supported by decidable subsets of the first order logic, which are known as description logics (DLs). Additionally, OWL syntax relies on RDF so that technology developed for RDF like triple stores and query languages can be directly applied to OWL. In this work we mainly focus on OWL-DL semantic descriptions. This kind of progress may be done with the help of Apriori algorithm. This apriori algorithm can be found the frequent item set of each and every document in the corpus.

\subsection{Removal of Non Useful Words}

In this process we have to do the process of avoidance of non-useful words. For this process we have to take the contribution of extensibility of existing lightweight ontologies into formal ontology. Ontology can be thought of as directed graphs consisting of concepts as nodes and relations as the edges between the nodes. A concept is essentially a mental symbol often realized by a corresponding lexical representation (i.e., natural language name). For instance, the concept "food" denotes the set of all substances that can be consumed for nutrition or pleasure. In Information Science, an ontology is a "formal, explicit specification of a shared conceptualisation". This definition imposes the requirement that the names of concepts and how the concepts are related to one another have to be explicitly expressed and represented using formal languages, such as Web Ontology Language (OWL). An important benefit of a formal representation is the ability to specify axioms for reasoning in order to determine validity and to define constraints in ontologies. Moreover, a formal ontology is natural language independent or in other words, does not contain lexical knowledge.

The output of our process is terms in a controlled vocabulary should be defined in a way to minimize or avoid ambiguity and redundancy. A taxonomy, on the other hand, is a controlled vocabulary organized into a hierarchical or parent-child structure. A thesaurus is similar to a taxonomy, with the addition of more relationships beyond hierarchical.

Here, we have the non-useful words and also the redundant words in our selected semantic web data document. After removing these words the structure of tree can be represented in our output window. It can be shown the complete process results of each and every document in our corpus. Because here, we were chosen the corpus of lonely planet. This lonely planet can contain the details of tourism.

\section{EXPERIMENTS}

In this allotment, we achieve the inclusive set of experiments to examine the performance of the proposed method of ARSRE framework by comparing it into the state-of-art method. The proposed ARSRE technique consists of three processes. There are Association Rule Mining, Removal of Redundant Words and Learning process. This technique can provide improved results of semantic relation extraction. Using this proposed technique we have to avoid the redundant and also non useful words in progress. The semantic relation extraction results and some other dataset results are should be given below.

\subsection{Experimental Testbed}

In our experimental testbed we have to use the input of lonely corpus planet dataset. This dataset having the fields of countries, cities, cultures, organization, person and etc. This dataset is taken fromthe http://www.lonelyplanet.com/destinations. The reason for choosing the dataset is to scrutinize the presentation of semantic relation extraction for more consideration. This dataset has 
International Journal of Web \& Semantic Technology (IJWesT) Vol.5, No.4, October 2014

different customs of input and details. But we are choosing only the countries and cities related details and we processed those details only limit to other properties in the dataset. Thus the dataset is suitable for our proposed ARSRE technique.

\subsection{Comparison}

In our experiment, we compared the proposed ARSRE technique with state-of-art method like CIV Based Semantic Relation Extraction using Markov Logic Network (CIVSRE). The previous CIVSRE method contains the processes of first-order logic predicates and finding inference and learning weight based semantic relation extraction. Our proposed technique also does the same processes first-order logic predicates and finding inference and learning weight semantic extraction with some slight changes. The changes can be highlighted first in semantic relation extraction process to find the frequent items between the relationships we have to use Association Rule Mining. Second, to extensibility of existing lightweight ontologies to formal ontology. The input dataset can be loaded into the application. The dataset could be processed by the GATE tool. This GATE tool can take the data's in corpus and processed by some processor of this tool and generate the corrected information of dataset. This input also enters into the progress for semantic relation extraction. The processed dataset will be used to construct the first-order logic predicates. For constructing the predicates, we have to use knowledge base (KB). This is mainly generated from the formulas of first order. These all are constructed with the help of type of symbols. Symbols consist of constants, variables, functions and predicates. The knowledge base formulas can be of two kinds of partitions. There are refutation and conjunctive normal form (CNF). A KB in clausal form is a conjunction of clauses, a clause being a disjunction of literals. Every KB in first-order logic can be converted to clausal form using a mechanical sequence of steps. Clausal form is used in resolution, a sound and refutation-complete inference procedure for first-order logic.

After performing this first-order logic we have to do some kind of building operations. First we build mln file, next build training data file and build the test files in the corpus. Here after do the process of identifying inference values and also weight learning. Our proposed technique can be producing the values of number of hidden predicates, number of formulas, evidence atoms and also optimal solution. Then next, process we were done in this extraction process is to find the frequent itemset relationship based upon the words. For that purpose we had to use association rule mining. According to that methodology every word in the dataset we have to know the relationship and also frequent items. Because frequent items are main thing in our proposed technique to find the relationship. Mining association rules basically consists of two portions first compute the frequent item sets respect to the minimum support, second one is generate rules from the frequent item sets according to the minimum confidence. As the number of frequent sets can be very large, closed and maximal item sets can be generated instead. Here, we use apriori algorithm to find the frequent item sets.

The final process of our proposed technique is to avoid non-useful words. Non-useful word means the word which is presented in the input file those are present meaningless. Those meaningless words are to be removed. For removal process we ensure the technology of conversion of lightweight ontology to formal ontology. To performing this step first we select all non useful words and avoid those words. Ontologies are fundamental to the success of the Semantic Web, as they enable software agents to exchange, share, and reuse. For the semantic web to function computers must have access to structured collections of information and sets of inference rules that they can use to conduct automated reasoning. For this reason, the majority of ontology learning systems out there that claim to learn ontologies are in fact, creating lightweight ontologies. At the moment, lightweight ontologies appear to be the most common type of ontologies in a variety of Semantic Web applications. 


\begin{tabular}{|l|l|l|}
\hline Technique & CIVSRE & ARSRE \\
\hline Precision & 119.85 & 233.33 \\
\hline Recall & 32 & 44.142 \\
\hline F1-Measure & 4,895 & 7,424 \\
\hline
\end{tabular}

Table 1 Comparison of Both Techniques

We also need to avoid the redundant words in the semantic extraction. According to the comparison results we have to know the efficiency level of semantic relation extraction. The efficiency of proposed and previous methods are calculated from the above table.

\subsection{Experimental Results}

This experiment seeks to study and organize the concept of semantic relation extraction into the ontology learning. Figure 3 shows the Precision, Recall and F1-Measures for the proposed technique. The ARSRE technique was evaluated by comparing its output with a CIVSRE. For this purpose we use the data set of Lonely Planet corpus for performing the semantic extraction task. Here we evaluate the relations from the dataset of Lonely Planet corpus. This evaluated result is the semantic relation extraction using the ARSRE technique. This ARSRE technique is based on the Markov Logic Networks, which is performed by the Alchemy software packages, and also performed by association rule mining.

The ARSRE technique consists of the steps of first-order logic predicates and finding inference and learning weight based semantic relation extraction. Here the construction of predicates mainly focuses the Knowledge Base of the formulas. According to the knowledge base we have to construct the first-order logic predicates. After that we have to build the .mln file, build training file and also build the test file. Then next, we have to find the inference and weight learning of the contents, this is done with the help of Markov Logic Network. These are performed by the same method taken from the state-of-art method. Then the process include in our proposed technique is to avoid the non useful words. For that purpose we have to select the non-useful words and avoid the redundant words in the scenario and also to make the tree structure using thesaurus. These all processes are covered by the ARSRE techniques.

The experiment results can be compared with the technique of CIVSRE and ARSRE. The results can be varying with the parameter of accuracy based on relationship. Our process should produce more accuracy when compared to the preceding technique. The accuracy can be calculated with the help of the values of precision, recall and alsoF1 measure. Our proposed ARSRE technique can have the best precision, recall and also F1 measure. According to those values the accuracy of every technique shall be calculated. 
International Journal of Web \& Semantic Technology (IJWesT) Vol.5, No.4, October 2014

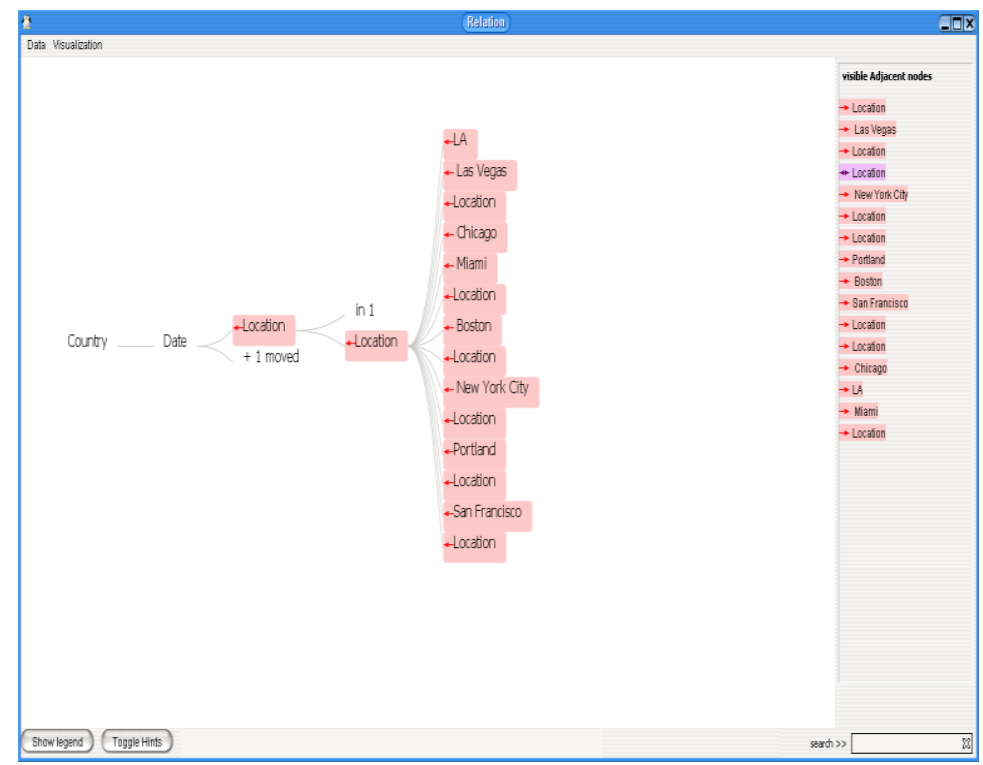

Figure 3 Lonely Planet Corpus Relationship Results

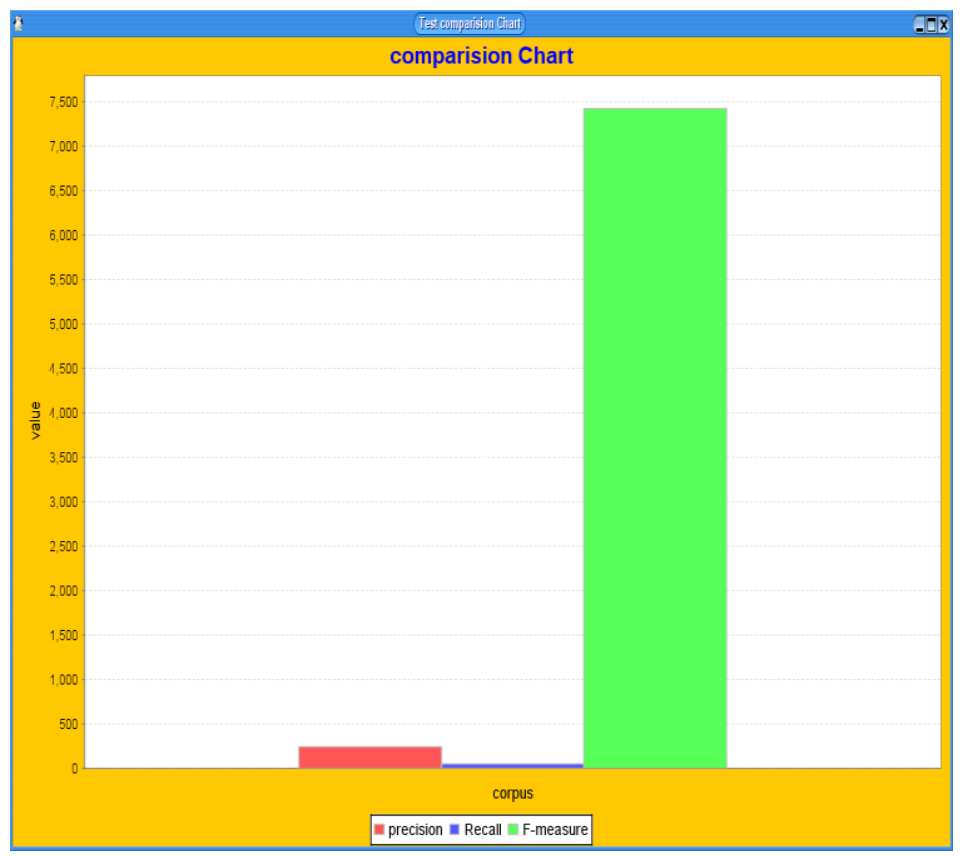

Figure 4 Lonely planet corpus experimental results

\section{Conclusion}

This paper presented a framework of ARSRE for semantic relation extraction which applies Markov Logic Networks for predicting the predicates, first order logic formula and also inference and weight learning. While a matter between efficiency and accuracy we first proposed deterministic ARSRE technique by means of the first-order logic predicates and semantic relation extraction. To implementing this ARSRE technique we have to use the dataset of lonely planet corpus. The dataset having the details of tourism countries, cities and temples like that. According to the dataset we take the input of words and further processes are carried out by techniques. First process of our semantic relation extraction is prediction of first-order logic formula. This process 
encourages the method of knowledge base. According to this knowledge base we have to construct the predicates. Because it might be used the knowledge base formula symbols. The symbols are carried out by many parts. There are constants, variables, functions and predicates. A term is any expression representing an object in the domain. It can be a constant, a variable, or a function applied to a tuple of terms. After that we have to build some kind of files such as .mln file, training file and also test files. Second process is find inference and weight learning we use MLN method to learning the weight of words. For that purpose we can use the simple weight learning method to produce the good results. The main progress include in that is to find the inference values. For finding the inference we could use alchemy process. It may be the software to produce the optimized values of every word in the corpus. Alchemy Packages also used for implement this identification process. Alchemy packages are used for make the perfect inference process. For implementing all this performance, we use the dataset of Lonely Planet. The final process of our ARSRE technique is the semantic relation extraction. In that state, need to do the efficient way of semantic relation extraction. For this reason we use Association Rule Mining for finding frequent items between the relationships of every input data. Performing this kind of process everyone use the Apriori algorithm and phrase based semantic relation identification processes. After performing that this proposed technique does the progress of avoidance of nonuseful words. This could be performed with the help of ontology learning (OWL). Using this we can convert the extensibility of lightweight ontology into formal representation. An important benefit of a formal representation is the ability to specify axioms for reasoning in order to determine validity and to define constraints in ontologies. Moreover, a formal ontology is natural language independent or, in other words, does not contain lexical knowledge. Our ARSRE technique can do the process of association rule mining based semantic relation extraction. It could produce the efficient way of finding frequent item set between the relationship of every words.

\section{FUTURE ENHANCEMENT}

In our paper we present the process of semantic relations methodology named as ARSRE. This technique should provide the results of semantic relation efficiently. Association Rule Mining could be used to produce the relationships between the frequent items. Here, we could also did the process of selecting the non-useful words and avoid these words and make the tree structured results. This technique could be used to produce the better efficiency of semantic relations between concepts and methods. In future, to improve the ontology learning progress we have to move onto the process of axiom learning. Axiom learning will be based on rules come from the semantic relation. For axiom learning we use DRIC. The process of DRIC is to be discovery of inference rules from text. Using this process we have to make the tree structure of the resulted words. Next, we decide to do the process of ontology population. In ontology population mainly will be useful for knowledge acquisition. Knowledge Acquisition activities that transfer data or text into instance data. To open resultant instant data using Protégé tools. This ontology population is to analyze the population in semi automatically. These ideas are decided to implement in the future.

\section{REFERENCES}

[1] Hassan Khosravi," Discriminative Strucure and parameter learning for Markov Logic Networks", In Proceedings of the 25th International Conference on Machine Learning (ICML), Helsinki, Finland, July 2008.

[2] Domingos and Richardson. 2007. Markov Logic: A Unifying Framework for Statistical Relational Learning. Introduction to Statistical Relational Learning (pp. 339 - 371). Cambridge, MA: MIT Press. 
International Journal of Web \& Semantic Technology (IJWesT) Vol.5, No.4, October 2014

[3] M. Richardson, P. Domingos. Markov logic networks. Machine Learning, 2006, 62(1\{2): 107 136.

[4] Dellschaft, K., Staab, S.: On how to perform a gold standard based evaluation of ontology learning. In: Proceedings of ISWC-2006 International Semantic Web Conference, 2006.

[5] Dr.V.Karthikeyani and K.Karthikeyan, "Migrate Web Documents into Web Data," Electronics Computer Technology (ICECT) 3rd International Conference, 2011, Vol. 5, pp. 249 - 253.

[6] Athanasios Tegos, Vangelis Karkaletsis, and Alexandros Potamianos, "Learning of Semantic Relations between Ontology Concepts using Statistical Techniques”, proceeding at Technical University of Crete, july 27, 2012.

[7] Thomas Hofmann, "Probabilistic Latent Semantic Analysis," In Proc. of Uncertainty in Artificial Intelligence, UAI'99, 1999, pp.289-296.

[8] Karthikeyan, K and Dr.V.Karthikeyani, “Understanding text using Anaphora Resolution”, Pattern Recognition, Informatics and Mobile Engineering (PRIME) 2013 International Conference, 2013, pp- $346-350$.

[9] Wilson wong, wei liu and mohammed bennamoun, "Ontology Learning from Text: A Look Back and into the Future," ACM Comput. Surv. 44, 4, August 2012, 36 pages.

[10] Paul Buitelaar, Philipp Cimiano and Bernardo Magnini, "Ontology Learning from Text: An Overview," in Applications and Evaluation, 2005, pp. 3--12.

[11] Lucas Drumond and Rosario Girardi, "A Survey of Ontology Learning Procedures," WONTO, volume 427 of CEUR Workshop Proceedings, CEUR-WS.org, 2008.

[12] Lucas Drumond and Rosario Girardi, “An Experiment Using Markov Logic Networks to Extract Ontology Concepts from Text," in ACM Special Interest Group on Applied Computing, 2010, pp. 1354-1358.

[13] Stanley Kok, Parag Singla, Matthew Richardson and Pedro Domingos, "The Alchemy System for Statistical Relational AI: User Manual", proceeding at University of Washington, Department of Computer Science and Engineering, Aug 3, 2007.

[14] Zhen LI, Janyi GUO, Zhengtao, Yantuan XIAN, Xin YAN and Sichao WEI, "The CIV Semantic Relations Extraction based on Markov Logic Networks", Kunming University of Science and Technology, proceedings of the Journal of Computational Information Systems 2013.

[15] A.Matthew Richardson and Pedro Domingos, "Markov Logic Networks," 2006, vol. 6, pp. 1-44.

[16] Fei Wu, Daniel S. Weld, “Automatically Refining the Wikipedia Infobox Ontology", 17th international conference on World Wide Web, pp- 635-644, 2008.

[17] Quang-Thang DINH, Christel Vrain and Matthieu Exbrayat, "Generative Structure Learning for Markov Logic Network Based on Graph of Predicates", IJCAI'11 Proceedings of the TwentySecond international joint conference on Artificial Intelligence - Vol. 2, pp. 1249-1254.

[18] Xiangfeng Luo, Kai Yan and Xue Chen, "Automatic Discovery of Semantic Relations Based on Association Rule" journal of software, vol. 3, no. 8, november 2008.

[19] W. Kang, Z. F. Sui. Synchronization Extraction of Ontology Concept Instances and the Attributes Based onThe Weak Guidance Web. Journal of Chinese Information, 2010. 6: 54 - 59.

[20] Kaustubh Beedkar, Luciano Del Corro, Rainer Gemulla, "Fully Parallel Inference in Markov Logic Networks”, proceeding at Max-Planck-Institut für Informatik, vol.2, 2011, pp- 373-384 .

[21] Hilda Koopman, Dominique Sportiche and Edward Stabler, "An Introduction to Syntactic Analysis and Theory", 2013.

[22] Sandeepkumar Satpal, Sahely Bhadra, S Sundararajan, Rajeev Rastogi and Prithviraj Sen, "Web Information Extraction Using Markov Logic Networks", 17th ACM SIGKDD international conference on Knowledge discovery and data mining, 2011, pp- 1406-1414.

[23] Alexander Maedche and Steffen Staab, "Ontology Learning for the Semantic Web", Intelligent Systems, vol.16, pp- 72-79, 2011.

[24] M. Richardson, P. Domingos. Markov logic networks. Machine Learning, 2006, 62(1\{2): 107 136.

[25] Hilda Koopman, Dominique Sportiche and Edward Stabler, “An Introduction to Syntactic Analysis and Theory", Proceedings of the 14th conference on Computational linguistics, vol. 2, Jan. 1992, pp. 539-545. 
International Journal of Web \& Semantic Technology (IJWesT) Vol.5, No.4, October 2014

[26] Hoifung Poon Pedro Domingos, "Sound and Efficient Inference with Probabilistic and Deterministic Dependencies", AAAI'06 Proceedings of the 21st national conference on Artificial intelligence - Volume 1,pp. 458-463.

[27] K.Karthikeyan and Dr.V.Karthikeyani, "PROCEOL: Probabilistic Relational of Concept Extraction in Ontology Learning", Internation Review on Computers and Software, Vol.9, No.4, 2014.

[28] Hamish Chunningham, Yorick Wilks and Robert J.Gaizauskas, "A General Architecture for Text Engineering", proceeding at an institute of language, speech and hearing, 2012.

[29] M. Poesio, A. Almuhareb, Identifying Concept Attributes Using a Classifier, Proceedings of the ACL-SIGLEX Workshop on Deep Lexical Acquisition, Ann Arbor, 2005: 8 - 27.

[30] J. Y. Guo, Z. Li, Z. T. Yu, etc. Extraction of Ontology Concept Instance, Attribute, Attribute Values and Their Relationship [J]. Journal of Nanjing University (Natural Sciences), 2012(48): 1 7.

\section{Authors}

KARTHIKEYAN K was born in Tamil Nadu, India in $13^{\text {th }}$ February 1975 . He is working as Assistant Professor in Department of Information Technology at NPR Arts \& Science College, Natham, Tamil Nadu, India. He was received M.Sc degree in Information Technology from Bharathidasan University, Tamil Nadu, India in 2002, M.Phil degree from Madurai Kamraj University, Tamil Nadu, India in 2006. He has published 2 National and 2 International Conferences Papers and One International Journals and presented papers in National and International Conferences. He has 10 years teaching experience. His areas of Interests are Data Mining, Artificial Intelligence, Natural Language Processing, Data and Knowledge Engineering.

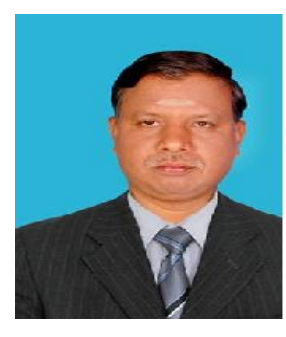

Dr. KARTHIKEYANI V was born in Tamil Nadu, India in $11^{\text {th }}$ June 1972. She is working as Assistant Professor in Department of Computer Science at Govt. Arts College, Rasipuram, Tamilnadu, India. She was awarded Doctoral degree from Periyar Universtiy, Salem, Tamilnadu, India. She has published 15 National and International Journals and presented several papers in International and National Conferences. She has 17 years of teaching experience. Her areas of interests are Image Processing, Computer Graphics, Multimedia, Data Mining and Web Mining. She is a life member in Computer Society of India (CSI), ISTE (Indian Society for Technical Education), ACM-CSTA (Computer Science Teacher Association) and various other International Computer Societies and organization for

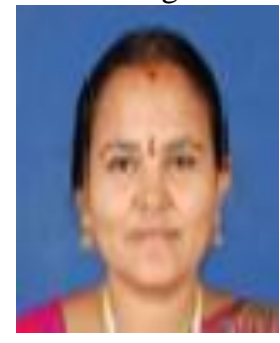
knowledge exchange and enhancement. 